\title{
Phospholipase $C_{\gamma} 1$ links inflammation and tumorigenesis in colitis-associated cancer
}

\author{
Kwang-Il Park ${ }^{1}$, Kwang-Youn Kim ${ }^{1}$, Tae Woo Oh${ }^{1}$, Du-Seock Kang ${ }^{2}$, Eung-Kyun \\ $\mathrm{Kim}^{3}$, Yong Ryoul Yang${ }^{2}$, Young-Kyo Seo ${ }^{2}$, Jin-Yeul Ma' ${ }^{1}$ and Pann-Ghill Suh ${ }^{2}$ \\ ${ }^{1}$ Korean Medicine (KM)-Application Center, Korea Institute of Oriental Medicine (KIOM), Cheomdan-ro 70, Dong-gu, Daegu \\ 41062, Republic of Korea \\ ${ }^{2}$ School of Life Sciences, Ulsan National Institute of Science and Technology (UNIST), UNIST-gil 50, Ulsan 44919, Republic \\ of Korea \\ ${ }^{3}$ Gene and Cell Therapy Research Center for Vessel-associated Diseases, Pusan National University, Yangsan 50612, Republic \\ of Korea
}

Correspondence to: Pann-Ghill Suh, email: pgsuh@unist.ac.kr

Keywords: phospholipase C yl; inflammation; colorectal cancer; intestinal barrier dysfunction; inflammatory bowel disease

Received: June 26, $2017 \quad$ Accepted: November 29, $2017 \quad$ Published: December 19, 2017

Copyright: Park et al. This is an open-access article distributed under the terms of the Creative Commons Attribution License 3.0 ( $C$ C BY 3.0), which permits unrestricted use, distribution, and reproduction in any medium, provided the original author and source are credited.

\section{ABSTRACT}

Colorectal cancer (CRC) is the third diagnosed cancer and the second leading cause of cancer-related deaths in the United States. Colorectal cancer is linked to inflammation and phospholipase CY1 (PLCY1) is associated with tumorigenesis and the development of colorectal cancer; however, evidence of mechanisms connecting them remains unclear. The tight junctions (TJ), as intercellular junctional complexes, have an important role for integrity of the epithelial barrier to regulate the cellular permeability. Here we found that PLCY1 regulated colitis and tumorigenesis in intestinal epithelial cells (IEC). To induce the colitis-associated cancer (CAC), we used the AOM/DSS model. Mice were sacrificed at 100 days (DSS three cycles) and 120 days (DSS one cycle). In a CAC model, we showed that the deletion of PLCY1 in IEC decreased the incidence of tumors by enhancing apoptosis and inhibiting proliferation during tumor development. Accordingly, the deletion of PLCY1 in IEC reduced colitisinduced epithelial inflammation via inhibition of pro-inflammatory cytokines and mediators. The PLCY1 pathway in IEC accelerated colitis-induced epithelial damage via regulation of $\mathrm{TJ}$ proteins. Conclusions: Our findings suggest that PLCY1 is a critical regulator of colitis and colorectal cancer and could further help in the development of therapy for colitis-associated cancer.

\section{INTRODUCTION}

Cancer comprises a large family of diseases that is characterized by abnormal cell growth and invasion of surrounding tissues. Cancer cells undergo modified cellular processes, such as cell proliferation, migration, and differentiation, using altered signaling pathways. Colorectal cancer (CRC) is the third most frequently diagnosed cancer and the second leading cause of cancer-related deaths in the United States. Various mutations have been associated with the development of CRC, including the Wnt signaling pathway, K-Ras, $\mathrm{p} 53$, and the transforming growth factor $\beta$ pathway $[1,2]$.
Cancer-associated inflammation, as a hallmark of cancer, is an important factor in the development of CRC; other environmental factors, such as epigenetic abnormalities and genetic mutations also play important roles in this process [3-5]. It has been reported that chronic inflammation is the leading cause of increased risk of several types of cancers including colon, liver, prostate, and breast cancer $[6,7]$. Patients with inflammatory bowel disease (IBD), such as Crohn's disease and ulcerative colitis, have a higher risk of developing CRC compared with healthy people. IBD, which is characterized by chronic inflammatory conditions of the gastrointestinal (GI) tract, results not only in 
intestinal epithelial barrier disruption but also in an influx of immune cells [8-10]. Tight junctions (TJs) of the intestinal epithelium play a pivotal role in the regulation of barrier function and protect the body from toxins, allergens, and pathogens in the intestine. IBD has been associated with an increased risk of CRC and the development of CRC between the stages of inflammation and carcinogenesis. This link between inflammation and the development of CRC has been extensively studied using mouse models of defects in the epithelial barrier in colitis and the formation of colorectal tumor. However, the contributing factors and underlying mechanisms have yet to be identified.

Phosphoinositide-specific phospholipase C (PLC) regulates cellular ligand-mediated signal transduction. PLC hydrolyzes phosphatidylinositol-4,5-bisphosphate (PIP2) into inositol-1,4, 5-triphosphate (IP3) and diacylglycerol (DAG). PLCs are activated by various extracellular ligands, such as growth factors, hormones, cytokines, and lipids. Several studies have shown that the activation of PLCs is associated with tumorigenesis and/or the progression of metastasis, such as migration, proliferation, growth, inflammation, angiogenesis, and the rearrangement of the actin cytoskeleton. As one of 13 mammalian PLC isozymes, PLC gamma 1 (PLC $\gamma 1$ ) is primarily activated by extracellular stimuli and is involved in tyrosine kinase signaling and the promotion of tumor cell growth and migration $[11,12]$. There is also evidence that PLC $\gamma 1$ plays a critical role in cell adhesion and is highly expressed in metastatic tumors [13]. A genetic deficiency in PLC $\gamma 1$ was shown to play an important role in metastasis and anti-apoptosis of human CRC, indicating that PLC $\gamma 1$ can function as a oncogene in vitro [14].

Despite the increasing number of studies seeking to identify the possible link between PLC $\gamma 1$ and tumorigenesis, the pathogenic role of PLC $\gamma 1$ in inflammation-related cancers, particularly colitisassociated cancer (CAC), has yet to be investigated. The present study is the first to use intestine-specific PLC $\gamma 1$ conditional knockout mice as an experimental model to investigate the role of PLC $\gamma 1$ in intestinal inflammation and CAC. Ablation of PLC $\gamma 1$ in intestinal epithelial cells (IECs) significantly ameliorated CAC progression. Our study sought to identify the role of PLC $\gamma 1$ in tumorigenesis under physiological conditions.

\section{RESULTS}

\section{Deletion of PLC $\gamma 1$ in IECs decreases the incidence of $\mathrm{CAC}$}

Azoxymethane (AOM) acts as a carcinogen via the formation of $\mathrm{O}_{6}$-methyl guanine [15]. AOM/dextran sulfate sodium salt (DSS) was shown to induce tumors in the colons of rodent (distal to middle segments) and is commonly used in experimental CRC animal models [16]. We generated PLC $\gamma 1$ IEC-specific knockout mice using Villin-Cre and PLC $\gamma 1$ alleles to investigate the effects of the PLC $\gamma 1$ deletion on IECs. We designed two experimental protocols to test the effects of inflammation on CAC. In the first study, 6-8 weekold mice were intraperitoneally (IP) injected with a single $10 \mathrm{mg} / \mathrm{kg}$-dose of AOM followed by one or three cycles of $2 \%$ DSS administered in the drinking water (Figure 1A and 1E). Repeated DSS administration, used to mimic IBD, was conducted to cause AOM-induced tumors [17]. PLC $\gamma 1$ conditional knockout mice and PLC $\gamma 1^{\mathrm{f} / \mathrm{f}}$ [wild type (WT)] littermates developed colon tumors, primarily in the distal to middle segments, using the AOM/DSS protocol (Figure 1B, 1D, 1F, $1 \mathrm{G}$ and $1 \mathrm{H}$ ), consistent with the localization of human colorectal tumors, the most severe consequence of DSS-induced colitis [18]. However, mice receiving DSS alone did not produce tumors during the experimental period (data not shown). When PLC $\gamma 1$ conditional knockout mice were subjected to three cycles of DSS, we observed a $50 \%$ decrease in the incidence of tumors, and the average tumor load was lower than that in WT mice. In addition, macroscopic tumors ( $>4 \mathrm{~mm}$ ) were detected only in WT mice (Figure 1C). Histological analyses showed more low- and high grade tumors in WT mice than in PLC $\gamma 1$ conditional knockout mice, but the relative proportion of low- versus high grade tumors was similar in both mouse groups. When mice were subjected to only one cycle of DSS (Figure 1E), we noticed that WT mice only manifested colon tumors, whereas no colon tumors were detected in PLC $\gamma 1$ conditional knockout mice (Figure $1 \mathrm{~F}$ and $1 \mathrm{H}$ ). In addition, the incidence of tumors in WT mice undergoing one cycle of DSS was reduced by approximately $50 \%$ compared with WT mice undergoing three cycles of DSS.

This result suggests that the difference between the incidence of tumors causing an inflammatory response and the frequency of DSS administration is due to the effects of PLC $\gamma 1$ on inflammatory responses caused by DSS and on both tumor initiation and development. Adenomatous polyposis coli (APC) or $\beta$-catenin gene mutations lead to increased tumor incidence through the stabilization of $\beta$-catenin and transcriptional activation with TCF-4, which play a pivotal role in CRC [19]. Genomic DNA from the tissue was isolated using laser capture microdissection and purified; exon 3 of the $\beta$-catenin gene was sequenced to look for mutations. We found that exon 3 of the $\beta$-catenin gene, which corresponds to a GSK3 $\beta$ phosphorylation sites, contained a serine-to-cysteine mutation in codon 33 in both WT and PLC $\gamma 1$ conditional knockout mice (Supplementary Figure 1). These results suggested that the severity of inflammation is associated with increased tumor incidences and that PLC $\gamma 1$ plays a pivotal role in tumor initiation and/or promotion. 


\section{Deletion of PLC $\gamma 1$ in IECs reduces proliferation and induces apoptosis in CAC}

Apoptosis is involved in tumor progression and maintenance. PLC $\gamma 1$ regulates apoptosis enzymatically by proteolytic cleavage $[20,21]$. We found that AOM/ DSS induces apoptosis more strongly in PLC $\gamma 1$ conditional knockout mice than in WT mice. As expected, PLC $\gamma 1$ was strongly expressed in the AOM/ DSS tumor model, whereas it was expressed at approximately normal levels in WT mice (Figure 2A).
Numbers of cleaved caspase-3 positive cells were decreased in WT mice compared to those in PLC $\gamma 1$ conditional knockout mice (Figure 2B). Analyses of apoptosis-related proteins revealed that the levels of the proapoptotic protein Bak and cleaved poly (ADPribose) polymerase (PARP) were significantly increased, whereas levels of phospho-AKT, phosphor-STAT3, and phospho-p65 were decreased in PLC $\gamma 1$ conditional knockout mice. In contrast, Bax and Bcl-XL were expressed at similar levels in WT and PLC $\gamma 1$ conditional knockout mice. The activation of c-Jun N-terminal
A

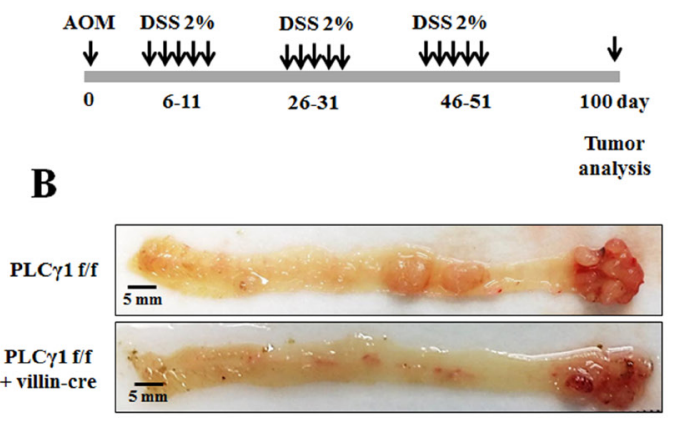

C

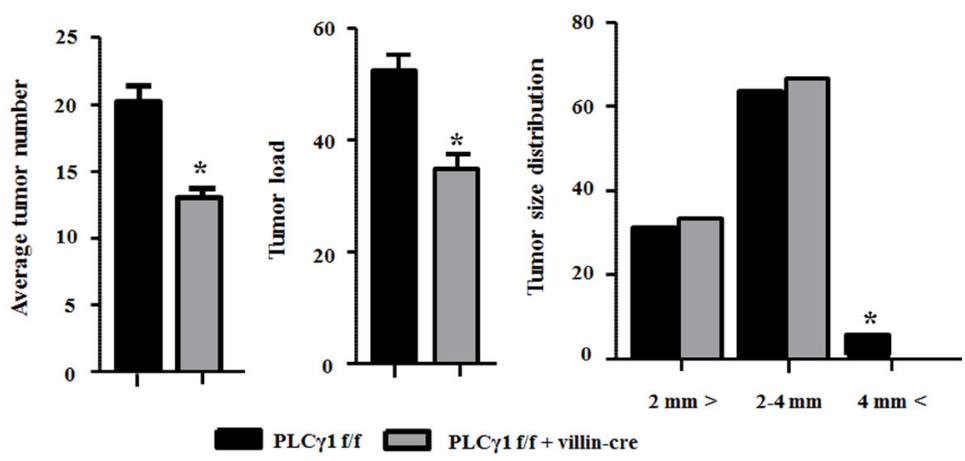

$\mathbf{E}$

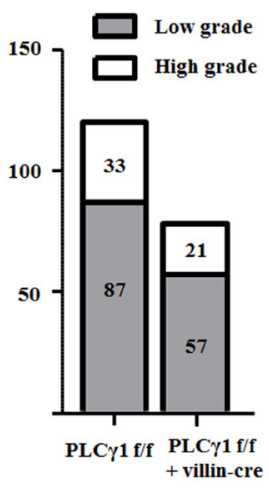

$\mathbf{F}$
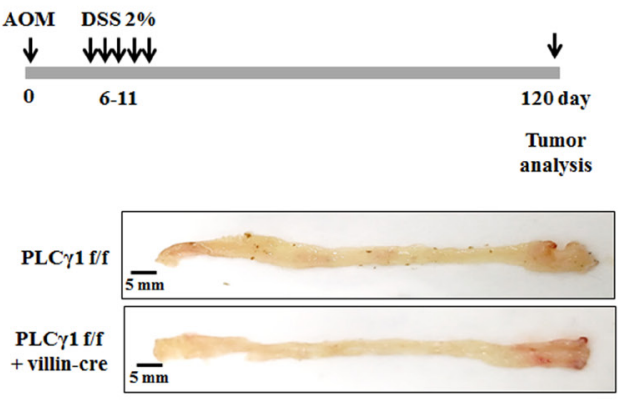

$\mathbf{H}$

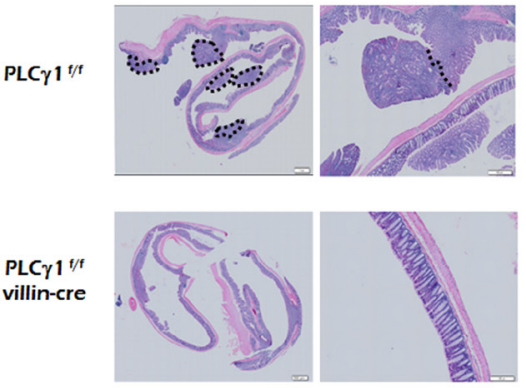

Figure 1: Deletion of PLC $\gamma 1$ in IECs decreases AOM/DSS-induced tumor incidence in colorectal ducts. (A) Design of the AOM/DSS protocol (DSS three cycles). (B) Representative images of colon tumors. (C) Average tumor number, load, and size distribution. Data represent the means \pm SEM $(n \geq 6) .{ }^{*} p<0.05$. (D) H\&E staining of tumors (E) Schematic representation of the AOM/DSS protocol (DSS one cycle). (F) Representative images of colon tumors. (G) Average tumor number, load, and size distribution. Data represent the means $\pm \operatorname{SEM}(n \geq 6)$. (H) H\&E staining of tumor morphology. 
kinase has been shown to induce apoptosis [22, 23]. However, the expression levels of mitogen-activated protein kinases were not changed (Figure 2C). We used terminal deoxynucleotidyl transferase-mediated dUTP nick- end- labeling (TUNEL) to investigate apoptotic responses in tumors from $\mathrm{WT}$ and PLC $\gamma 1$ conditional knockout mice (Figure 2D). In contrast to WT mice, TUNEL-positive cells were detected in tumors isolated from PLC $\gamma 1$ conditional knockout mice. The decrease in tumor incidence could explain the increase in apoptosis by the upregulation of Bak and cleaved PARP, and the downregulation of phospho-AKT, in tumors from PLC $\gamma 1$ conditional knockout mice. The proliferation of tumor cells plays a critical role in tumor development; previous studies showed that PLC $\gamma 1$ is essential for cell proliferation and differentiation $[24,25]$. We tested cell proliferation in colon tumors from AOM/DSS-treated mice. We detected dividing tumor cells using p-histone H3 immunohistochemistry (IHC) and found higher proliferation rates in tumors from WT mice compared to PLC $\gamma 1$ conditional knockout mice. Consistent with hematoxylin and eosin (H\&E) staining, the proliferation of tumor cells was much higher in WT mice than in PLC $\gamma 1$ conditional knockout mice during tumor progression and development (Figure 2D). These results suggest that the difference in tumor incidence caused by apoptosis and proliferation of tumor cells occurs through the activation of PLC $\gamma 1$.
A

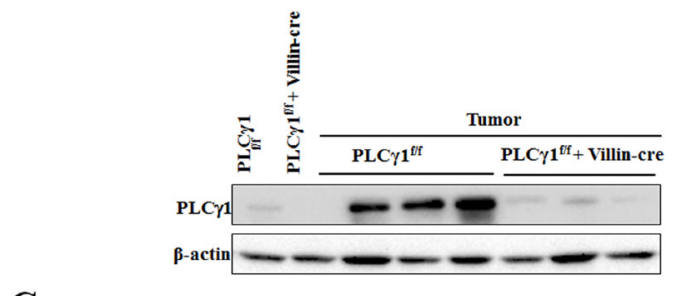

B

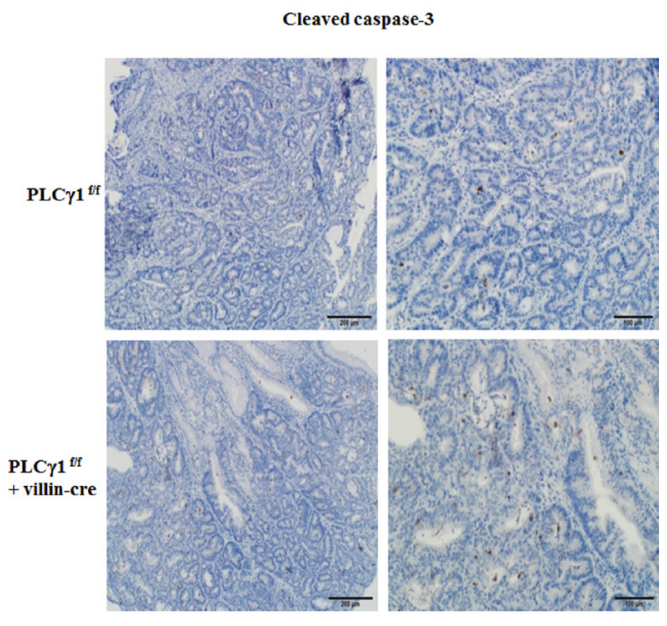

C

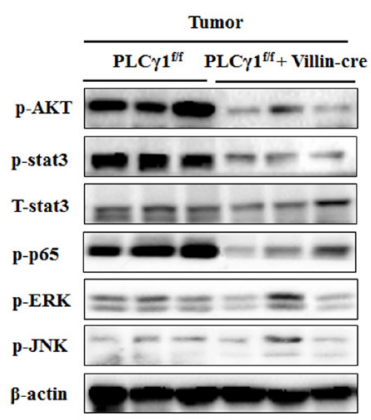

D

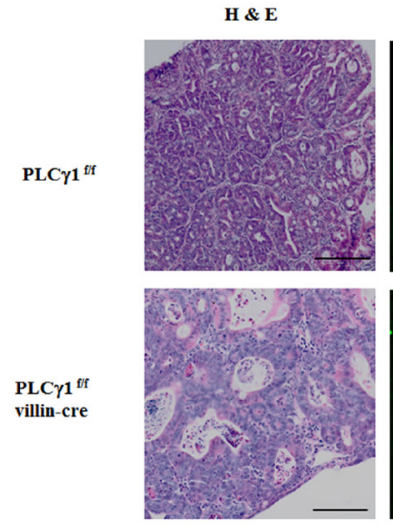

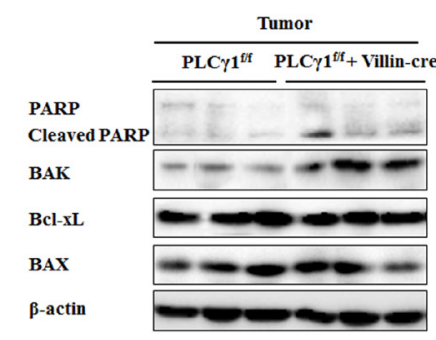

p-Histon h3
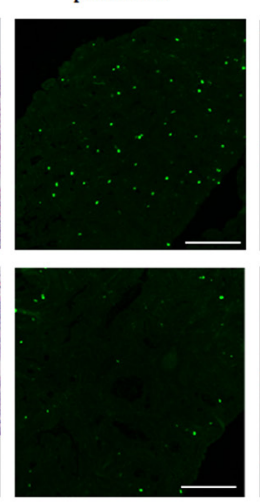
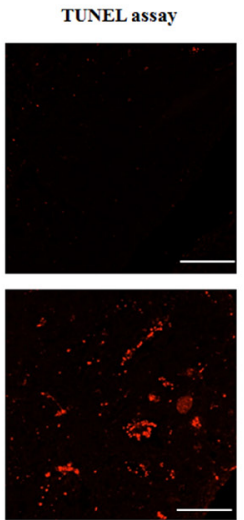

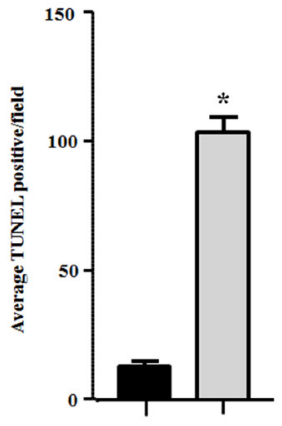

PLC $\gamma 1$ f/f $\square$ PLC 1 1 f/f + villin-cre

Figure 2: In the AOM/DSS model, deletion of PLC $\gamma 1$ in IECs decreases tumor incidence by increasing apoptosis and inhibiting proliferation. (A) Western blotting of PLC $\gamma 1$ in whole-colon lysates (colon). (B) Colon tumor tissues were obtained from $\mathrm{PLC} 1^{\mathrm{f} / \mathrm{f}}$ (WT) and PLC $\gamma 1$ conditional knockout mice, and samples were stained for cleaved caspase-3. (C) Colon tumor tissues were obtained from PLC $\gamma 1^{\mathrm{f} / \mathrm{f}}$ and PLC $\gamma 1$ conditional knockout mice, and samples were analyzed by western blotting (one mouse per lane) with the indicated antibodies. Data represent the means $\pm \operatorname{SEM}(n=3)$. (D) Colon tumor tissues were obtained from PLC $\gamma 1^{\mathrm{f} / \mathrm{f}}$ and PLC $\gamma 1$ conditional knockout mice, and samples were stained for H\&E staining, and p-Histone H3, and used for TUNEL assay. 


\section{IEC-specific deletion of PLC $\gamma 1$ prevents DSS- induced inflammation}

Although a recent study did not report any direct evidence for the effects of PLC $\gamma 1$ inhibition on attenuated colonic inflammation, we hypothesize that PLC $\gamma 1$ contributes to the inflammatory response, including via the NF- $\kappa B$ signaling pathway [26]. To assess whether PLC $\gamma 1$ may affect colonic inflammation, we applied the DSS-induced acute colitis model in PLC $\gamma 1$ conditional knockout mice and their littermate controls (Figure 3A). This method provides an indication of the mechanisms behind the initiation and/ or development of AOM/DSS-induced tumors [27]. After three days of DSS administration, WT mice lost more body weight than did PLC $\gamma 1$ conditional knockout mice (Figure 3B), which is an induction of the severity of DSS-induced colitis. Another indicator, DSS-induced shrinkage in colon length did not differ between PLC $\gamma 1$ conditional knockout mice and WT mice (Figure 3C). The expression of PLC $\gamma 1$ also did not differ between DSS-treated WT mice and PLC $\gamma 1$ conditional knockout mice (Figure 3D). These results suggest that PLC $\gamma 1$ stimulates inflammation and IEC damage. To confirm this hypothesis, mice were provided DSS and analyzed three, five, and eight days. At each time point, the colons of WT mice presented mild to severe colonic inflammation and a disrupted intestinal architecture, whereas the colons of PLC $\gamma 1$ conditional knockout mice presented moderate to mild inflammation and a maintained intestinal architecture (Figure 4). In particular, WT mice had more infiltrating cells and fewer goblet cells compared to PLC $\gamma 1$ conditional knockout mice after eight days (Figure 4). We also found that DSS-treated WT mice showed significantly more damage to IECs in the distal-to middle segments of the colon compared to PLC $\gamma 1$ conditional knockout mice (data not shown).

\section{Deletion of PLC $\gamma 1$ in IECs reduces DSS-induced inflammation}

$\mathrm{AOM}$ and various alkylating agents induce rapid p53-dependent apoptosis of IECs by stimulating DNA damage [22]. We investigated whether AOM may further enhance apoptosis in PLC $\gamma 1$ conditional knockout mice. However, AOM alone did not contribute to apoptosis via DNA damage in PLC $\gamma 1$ conditional knockout mice (Supplementary Figure 2). These results suggest that the variability in tumor frequency between WT and PLC $\gamma 1$ conditional knockout mice was not caused by AOMinduced DNA damage or apoptosis.
A

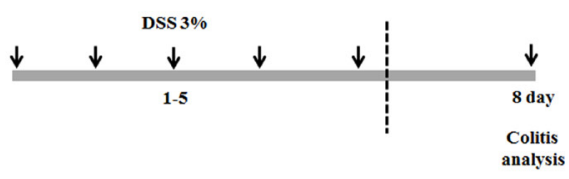

C

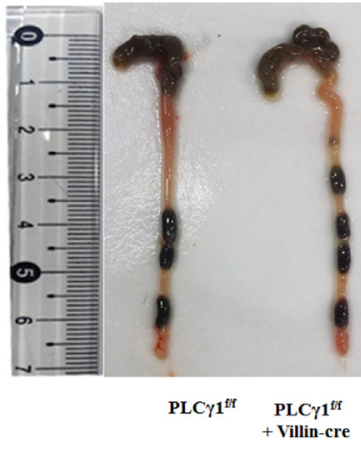

B

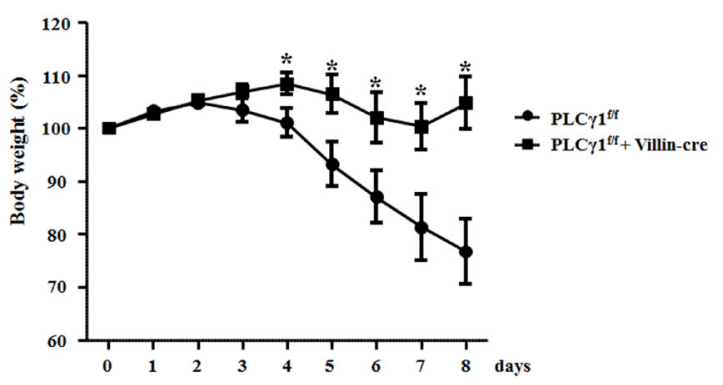

Figure 3: Deletion of PLC $\gamma 1$ in IECs decreases DSS-induced colitis. (A) Schematic representation of the acute colitis protocol. (B) DSS was administered in drinking water for five days, and body weight was recorded. Data are the means $\pm \operatorname{SEM}(n \geq 10),{ }^{*} p<0.05$. (C) PLC $\gamma 1^{\text {fff }}$ (WT) mice exhibit colon shortening after eight days of treatment with 3\% DSS compared with PLC $\gamma 1$ conditional knockout mice. Data represent the means $\pm \operatorname{SEM}(n \geq 10)$. ${ }^{*} p<0.05$. (D) Colon lysates were obtained from DSS-treated mice, and the expression of PLC $\gamma 1$ by western blotting (one mouse per lane) was analyzed. 
During colonic inflammation, cytokines and chemokines from infiltrating immune cells play crucial roles in intestinal tissues. We found that mRNA levels of cytokines, including interleukin (IL-) 6, tumor necrosis factor alpha, IL-1 $\beta$, and cyclooxygenase-2, were significantly increased in colons from DSS-treated PLC $\gamma 1$ conditional knockout mice compared to WT mice (Figure 5A). IL-6 levels increased more in WT mice than the other cytokines, because the PLC $\gamma 1$ pathway is linked to IL-6 signaling [28, 29]. The upregulation of IL-6 enhanced inflammation via the infiltration of immune cells by paracrine and/or autocrine mechanisms [30]. In addition, we detected higher expression levels of inflammation-mediated transcription factors such as IкB, nuclear factor (NF)- $\kappa$ B, and STAT3; resulting in a decrease in the phosphorylation of both STAT3 and NF- $\kappa$ B by the activation of $\mathrm{I} \kappa \mathrm{B}$ in PLC $\gamma 1$ conditional knockout mice (Figure 5B). Taken together, these results demonstrate that decreased tumor incidence in PLC $\gamma 1$ conditional knockout mice is due to reduced inflammation.

\section{Deletion of PLC $\gamma 1$ in IECs induces an intestinal barrier dysfunction after DSS treatment}

Disrupted epithelial barrier function has been associated with IBD and colon cancer [8, 9]. Damage to IECs leads to inflammation of the colonic mucosa as a result of intestinal barrier dysfunction in patients with ulcerative colitis [31]. Levels of IL-6 are increased in IBD patients, which modulated the expression of $\mathrm{TJ}$ proteins both in vivo and in vitro $[32,33]$. We identified upregulated IL-6 levels in WT mice compared with PLC $\gamma 1$ conditional knockout mice. We hypothesized that the DSS-induced enhancement of IEC damage and increasing inflammation in WT mice triggered intestinal barrier dysfunction. As TJs are comprised of cytoplasmic scaffolding proteins, such as
ZO, occludin, claudin, and dynamic structures [34], which play pivotal roles in TJ structure and function, and recent study found that PLC $\gamma$ inhibition enhanced epithelial barrier dysfunction via the regulation of TJ proteins [35], we chose to investigate the role of PLC $\gamma 1$ in intestinal barrier dysfunction by analyzing the expression of TJ proteins using IHC in DSS treated colon tissues. We found that the expression levels of TJ proteins were decreased in apical lesions of the intestinal epithelium and redistributed into colonic tissues after 5 days of DSS treatment in WT mice (Figure 6A) compared to the finding that TJ proteins were maintained in PLC $\gamma 1$ conditional knockout mice (Figure 6B). A difference in TJ proteins was observed after eight days of DSS exposure (Figure 6C). We also detected the expression of zonula occludens-1 (ZO-1) and protein kinase $\mathrm{C}$ alpha $(\mathrm{PKC} \alpha$ ) by western blotting. PKC activation regulates paracellular permeability via the activation of myosin light- chain kinase and the redistribution of ZO-1 [36]. We identified PKC $\alpha$ activation and ZO-1 downregulation in the colons of DSS-induced WT mice, and the mRNA levels of ZO-1 and occludin were significantly decreased (Figure 6C). In addition, mRNA levels and protein expression of ZO-1 and occludin were significantly decreased in colon tumors from AOM/ DSS-treated WT mice Supplementary Figure 3A and 3C), but mRNA levels of IL-6 did not change (Supplementary Figure 3B). Collectively, these results indicate that a reduction in inflammation after DSS treatment in PLC $\gamma 1$ conditional knockout mice is due to reduced intestinal barrier dysfunction via the inhibition of the PKC pathway and protection of TJ proteins.

\section{DISCUSSION}

CRC is one of the most common malignant tumors [37]. Several factors have been causally associated with
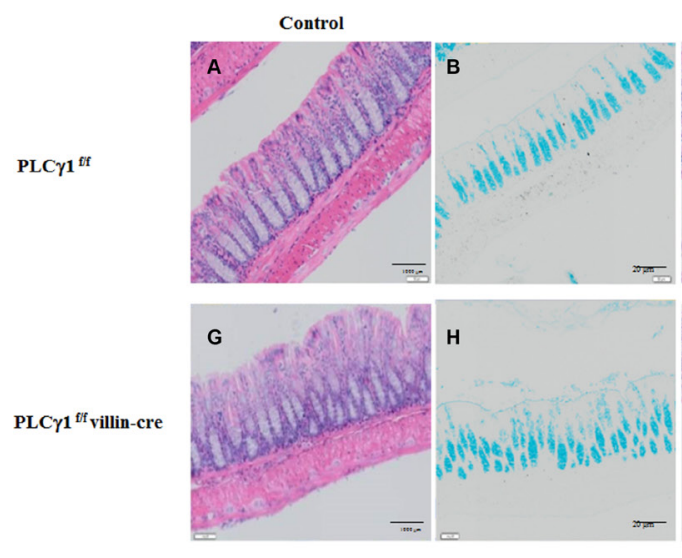
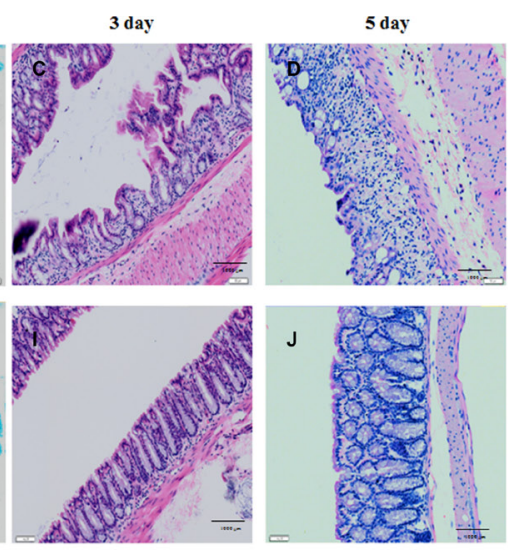
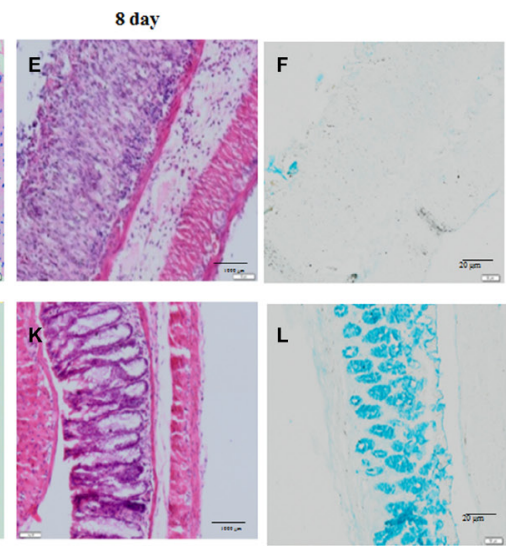

Figure 4: Histological changes in mice with DSS-induced colitis. Decreased susceptibility of PLC $\gamma 1$ conditional knockout mice treated with DSS to colitis. Colons of PLC $\gamma 1^{\mathrm{f} / \mathrm{f}}(\mathrm{WT})$ and PLC $\gamma 1$ conditional knockout mice were analyzed by H\&E staining three, five, and eight days after 3\% DSS exposure, and goblet cells were analyzed by Alcian Blue staining eight days after 3\% DSS exposure. (A, G) control H\&E staining. (C, I) 3 days H\&E staining. (D, J) 5 days H\&E staining. (E, K) 8 days H\&E staining. (B, H) control Alcian Blue staining. (F, L) 8 days Alcian Blue staining. 
A

IL-6

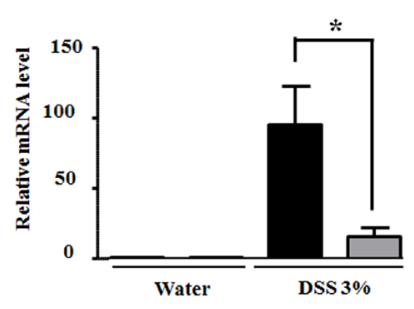

IL-1ß

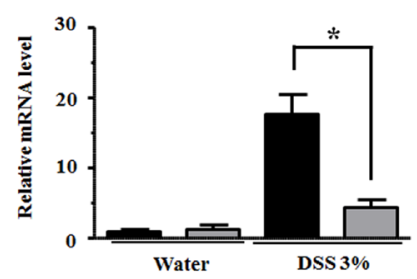

PLC $\gamma 1 \mathrm{f} / \mathrm{f}$

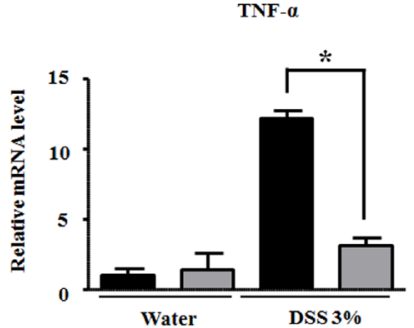

COX-2

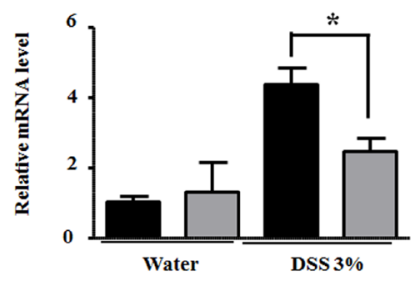

B

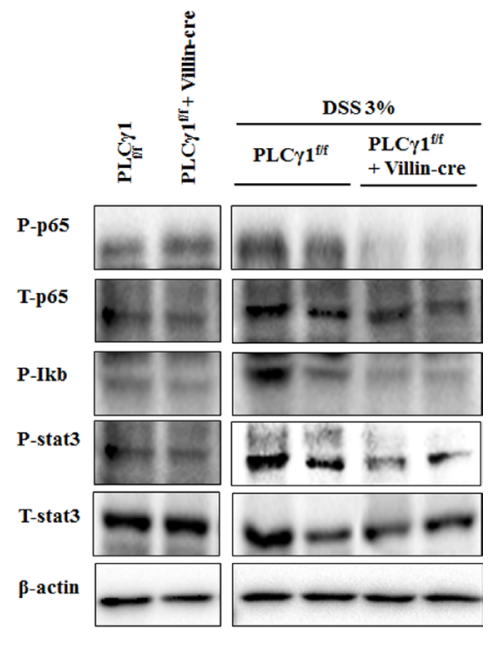

Figure 5: Deletion of PLC $\boldsymbol{\gamma} 1$ in IECs reduces DSS-induced inflammation. (A) Relative mRNA expression levels of the indicated genes in mouse colons were analyzed by qRT-PCR. Data are the means \pm SEM $(n \geq 10),{ }^{*} p<0.05$. (B) IECs were isolated from PLC $\gamma 1^{\mathrm{t} / \mathrm{f}}(\mathrm{WT})$ and PLC $\gamma 1$ conditional knockout mice after DSS exposure, and samples were analyzed by western blotting (one mouse per lane) with the indicated antibodies.

A

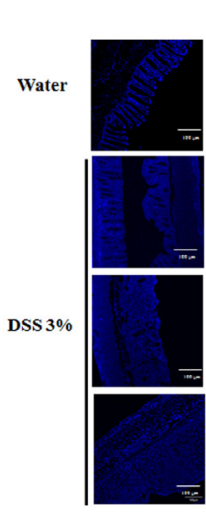

DAPI
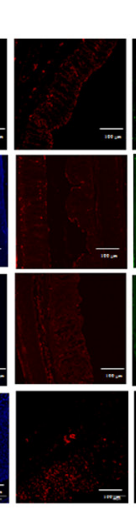

ZO-1
PLC- $\boldsymbol{1}^{\mathrm{ff}}$
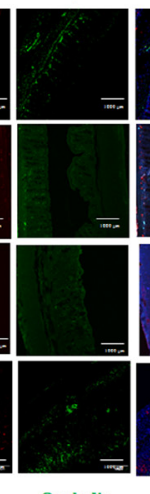

Occludin
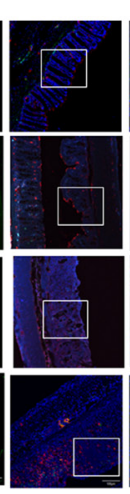

Merge 20x
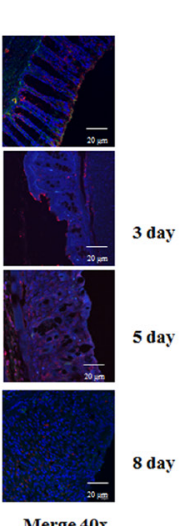

Merge 40x
B

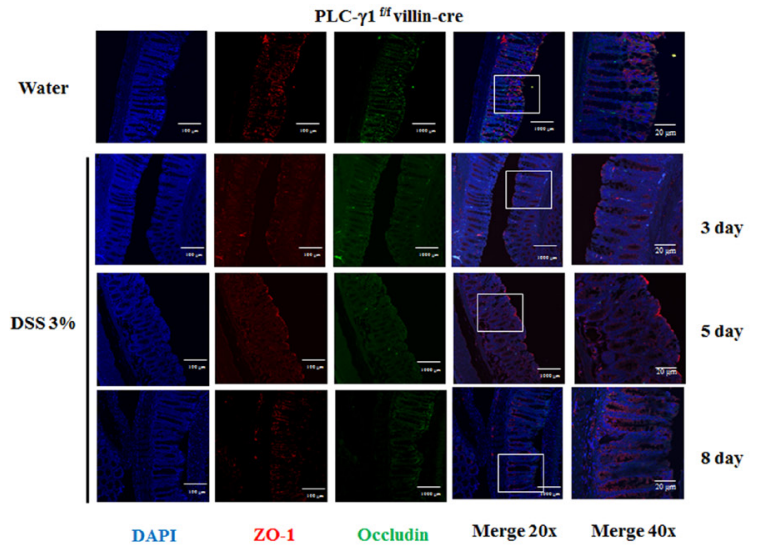

C
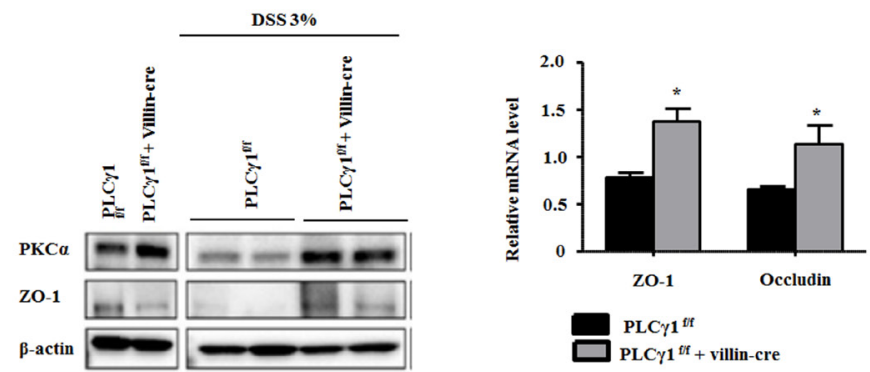

Figure 6: Deletion of PLC $\gamma 1$ in IECs increases intestinal barrier dysfunction. (A and B) DSS-induced IECs were obtained from PLC $\gamma 1^{\text {fff }}$ (WT) and PLC $\gamma 1$ conditional knockout mice at three, five, and eight days, and samples were stained by IHC with the indicated antibodies. (C) Colon lysates were analyzed by western blotting and qRT-PCR. Data are the means $\pm \operatorname{SEM}(n \geq 10),{ }^{*} p<0.05$. 
the development of CRC [38]. This work was designed to investigate whether the deletion of PLC $\gamma 1$ in IECs could reduce colitis-associated tumor incidence (CATI) through the induction of apoptosis.

In $\mathrm{CAC}$, the initial evidence for the functional relevance of PLC $\gamma 1$ signaling was provided in a preclinical trial that demonstrated that many of these targets are associated with cancer initiation and progression [17]. Our data indicate that tumor progression depends on the frequency of DSS exposure, suggesting that inflammation is associated with PLC $\gamma 1$ activation.

PLC hydrolyzes PIP2 into IP3 and DAG via IP3induced calcium release, and IP3 modulated DAG activates PKC [39]. Several studies have implicated PLC $\gamma 1$ as a regulator of cancer cell invasion and metastasis via multiple mechanisms. PLC $\gamma 1$ is a major transducer of growth factor and cytokine signaling. Downregulation of PLC $\gamma 1$ has been shown to inhibit human breast cancer cell-derived lung metastasis and progression in mice [40].

In this study, PLC $\gamma 1$ conditional knockout mice and WT littermates developed colon tumors, primarily in the distal to middle segments. Only WT mice developed macroscopic tumors $(>4 \mathrm{~mm})$ with a higher average tumor load and tumor number than in PLC $\gamma 1$ conditional knockout mice (Figure 1). Previous reports demonstrated that mutation at an important serine residue in codon 33 of $\beta$-catenin has a high oncogenic potential in AOM/ DSS-induced colon adenocarcinomas [41, 42]. Therefore, we confirmed the presence of this mutation in our mice and investigated its role in the mechanism of AOM/DSSinduced colon cancer. Our results were consistent with those of previous reports $[41,42]$. The mutation patterns in exon 3 of $\beta$-catenin were similar in both WT and PLC $\gamma 1$ knockout mice. These results demonstrate that AOM/DSSinduces cancer using the same mechanism in both mouse groups.

AOM/DSS-treated mice consistently develop metastases; however, the absence of a control genetic mutation that can be activated by a carcinogen makes this model system of questionable utility [16]. PI(3)K $\gamma^{-1}$ and $\mathrm{Apc}^{1638 \mathrm{~N} /+}$ mutants have been reported to develop colonic adenocarcinomas that metastasize to the lymph nodes and liver, which are common metastatic sites of human CRCs [16]. Activated STAT3 has been shown to be increased in CRC patients [43]; its activation induces certain antiapoptotic agents, such as Bcl-2 and Bcl-xl, which in turn increase CRC cell survival, proliferation, incursion, and lymph node metastasis [44]. Other studies have shown a correlation between STAT3 and Bak, which is probably related to the accumulation of proapoptotic molecules as well as to mediators of cell survival and growth [45]. PLC $\gamma 1$ cleavage can be blocked by the overexpression of Bcl-2 and specific caspase inhibitors [20].

We examined whether PLC $\gamma 1$ plays a pivotal role in IECs and whether AOM/DSS induces apoptosis in PLC $\gamma 1$ conditional knockout mice. In the AOM/DSS model, the
PLC $\gamma 1$, Bak, and cleaved PARP levels were significantly increased in tumors isolated from WT mice, whereas cleaved caspase-3 levels were decreased; however, in PLC $\gamma 1$ conditional knockout mice, phospho-AKT, phospho-STAT3, and phospho-p65 were all decreased (Figure 2). Although results from a previous report indicated that the interaction between AKT and PLC $\gamma$ was involved in cell growth and migration, the extent of cross-talk between their regulatory mechanisms remain unclear [46]. However, it is well known that AKT must be phosphorylated to stimulate cell proliferation through the activated PLC $\gamma / \mathrm{PKC} \gamma / \mathrm{Src} / \mathrm{PI} 3 \mathrm{~K}$ pathway in epidermal growth factor stimulated conjunctival goblet cells [40].

Recently, major proinflammatory pathways have been implicated in inflammation-associated tumor development. The carcinogenic potential of AOM is markedly increased by chronic inflammation, such as that induced by repeated cycles of DSS treatment. Furthermore, it has been reported that the IL-6/STAT3 signaling pathway plays an important role in AOM/DSSinduced murine CAC [47]. To investigate the effects of PLC $\gamma 1$ on CAC tumorigenesis, particularly on the IL-6/ STAT3 signaling pathway in AOM/DSS-treated mice, a deletion mutant of PLC $\gamma 1$ was used in a tumorigenic model of CAC and was shown to prevent inflammation by inhibiting the phosphorylation of STAT3 on Tyr705 [48]. Here, we showed that the expression levels of STAT3 phosphorylation were higher in WT mice than PLC $\gamma 1$ conditional knockout mice, leading to much higher tumor proliferation and inflammatory responses in the WT mice. As shown previously, the NF- $\kappa \mathrm{B}$-dependent tumor growth factor released by IECs could be IL-6, which plays an important role in proliferation [49] and can activates STAT3. Previous reports showed that DSS administration is well established to induce colitis, but removal of DSS leads to complete recovery of colitis to normalcy in 4-5 days [50]. IL-6 plays important roles in tumor initiation via PLC $\gamma 1$-mediated inflammatory response in colitis environment, and also after recovered colitis by removal of DSS, crosstalk between stat-3 and PLC $\gamma 1$ is involved in tumor progression and development through apoptosis and proliferation. This result could elucidate the role of PLC $\gamma 1$ in the suppression of the IL-6/STAT3 pathway; and provide further insight into the critical mechanisms involved (Figures 2C and 5).

The cellular localization of most PLCs is primarily cytosolic in the resting state; following receptor activation, PLCs are transiently recruited to the cell membrane. PLC-dependent pathways are postulated to be involved in the regulation of TJS [51]. In general, TJ formation is essential for early embryonic development, and PLCs may plays crucial roles in such developmental processes [52], a hypothesis supported by the developmental defects observed in various knockout mice lacking different PLC isozymes. PLC $\gamma$ is known to contain SH3 domains that bind proline-rich motifs on target proteins; interestingly, 
$\mathrm{ZO}$ proteins contain proline-rich sequences that could bind and localize PLC $\gamma$ to TJs [53]. Although no reports have described the colocalization of PLC $\gamma$ and TJs, PLC isozymes have been reported to colocalize with cortical actin filaments. Recently, PLC $\gamma 1$ was detected in human $\mathrm{T}$ cells as a phosphoprotein [54]. CD3 activation of $\mathrm{T}$ cells was shown to cause tyrosine phosphorylation of PLC $\gamma 1$, which is associated with a marked increase in PLC activity that accounts for the activation and translocation of PKC $\alpha$ and results in low expression levels of ZO-1 and claudin-2, thereby opening TJs in the epithelium [55]. Our data indicate that the reduction in inflammation after DSS treatment of PLC $\gamma 1$ conditional knockout mice is due to reduced intestinal barrier dysfunction via $\mathrm{PKC}$ pathway blockade and protection of TJ proteins via $\mathrm{PKC} \alpha$ (Figure 6).

Our data show that when PLC $\gamma 1$ is lacking in IECs, cancer incidence is reduced. It is possible that tumor incidence depends on the frequency of DSS exposure and is associated with a PLC $\gamma 1$-stimulated inflammatory response that affects tumor expression. Our study suggests that PLC $\gamma 1$ signaling may be a good target for therapies to reduce CATI. Indeed, PLC $\gamma 1$ is known to play important normal physiological roles, such as cell survival; and proliferation, as well as roles in cancer metastasis, such as angiogenesis and vascular permeability. The link between PLC $\gamma 1$ activity and the IL-6/STAT3 signaling pathway could account for the transcription of many important cancer-related genes. Although we have shown that the knockdown of PLC $\gamma 1$ induces apoptosis in the AOM-DSS model by mechanisms involving the downregulation of the IL-6/STAT3 signaling pathway, we aimed to demonstrate that PLC $\gamma 1$ may be an effective treatment for CRC by blocking the IL-6/STAT3 signaling. Studies of the function of PLC $\gamma 1$ are needed to elucidate potential novel targets for therapeutic intervention and provide new insights into the extent of PLC $\gamma 1$ participation in CATI.

\section{MATERIALS AND METHODS}

\section{Animals and induction of colitis and CAC}

PLC $\gamma 1^{\mathrm{f} / \mathrm{f}}(\mathrm{WT})$ and PLC $\gamma 1$ conditional knockout mice were generated with Villin-Cre mice [56]. The experiments were performed using 6-8 weeks old agematched mice in a C57BL/6 background. Their floxed littermates were used in all experiments as controls. Mice housing conditions and experimental protocols were approved by the Institutional Animal Care and Use Committee at Ulsan National Institute of Science and Technology. CAC was induced as described (Greten et al., 2004). Briefly, on day 1, mice were IP injected with $10 \mathrm{mg} / \mathrm{kg}$ AOM (Sigma- Aldrich) and maintained on regular diet and water for 5 days. After 5 days, mice received water with $2 \%$ (unless stated otherwise) DSS (dextran sulfate sodium, MP Biomedicals, molecular weight 35,000-50,000) for 5 days (DSS one cycle), followed by regular water for 14 days, and this protocol were performed during two further DSS treatment cycles (DSS three cycles). Mice were sacrificed at 100 days (DSS three cycles) and 120 days (DSS one cycle) after AOM injection, respectively. For acute colitis and inflammation studies, mice were administered 3\% DSS for 5 days, followed by regular water for 3 days and sacrificed at the indicated time points. Body weights were recorded during DSS treatment. Colon length was analyzed after removal from mice and flushed with cold phosphate-buffered saline (PBS), opened longitudinally, following which tumor number and size measurements were recorded. One half of the distal colon was used for Western blot and quantitative polymerase chain reaction (qPCR) analysis. The other half the colon tissues were fixed as "swissrolls" in 10\% formalin solution (Sigma, \#HT-501128) at room temperature overnight, and paraffin embedded.

\section{Histological analysis}

Paraffin-embedded colons were cut into $5 \mu \mathrm{m}$ thick sections, and serial sections were stained with $\mathrm{H} \& \mathrm{E}$ and Alcian Blue (for goblet cells). Using Scion Image for Windows (Zeiss), the colitis-severity and width of each tumor was measured and recorded.

\section{Immunoblotting}

Colon tissues were rinsed with ice-cold PBS and lysed using the radio-immunoprecipitation assay (RIPA) lysis buffer (Millipore Corporation, Billerica, MA, USA). Tissue lysates were centrifuged at 14,000 $\times \mathrm{g}$ for $30 \mathrm{~min}$. The supernatant was collected, and protein was measured using the BCA Protein Assay Kit. The proteins were separated by sodium dodecyl sulfate polyacrylamide gel electrophoresis (SDS-PAGE) gel and were electrophoretically transferred to polyvinylidene fluoride (PVDF) membrane (Millipore Corporation, Billerica, MA, USA). The membranes were blocked in 5\% skimmed milk in Thermo Scientific SuperBlock (TBS) containing 0.05\% Tween-20 (TBST) buffer for $1 \mathrm{~h}$, and then incubated with primary antibodies overnight at $4^{\circ} \mathrm{C}$. After washing in TBS-T buffer, the membranes were incubated with secondary antibodies for $1 \mathrm{~h}$ at room temperature. Protein bands were detected with Immobilon Western substrate (Millipore Corporation, Billerica, USA) and analyzed with the ChemiDoc Touch Imaging System (Bio-Rad, Hercules, CA, USA).

\section{Immunohistochemistry}

Sections of paraffin-embedded colon tissues were dewaxed in xylene, rehydrated, and subjected to antigen retrieval in $10 \mathrm{mM}$ citrate buffer. Sections were blocked for $30 \mathrm{~min}$ in $1 \%$ bovine serum albumin, $0.02 \%$ Triton $\mathrm{X}-100$, and 5\% normal goat serum (NGS). Serial sections 
were incubated with rabbit polyclonal ZO-1 antibody (1:200 dilution) and occludin antibody (1:200 dilution), followed by Cy5-conjugated goat anti-rabbit secondary antibody (Jackson ImmunoResearch Laboratories, Inc., West Grove, PA, USA). The sections were mounted using Vectashield with 4',6-diamidino-2-phenylindole (DAPI) (Vector Laboratories, Burlingame, CA, USA). Images from serial sections were acquired using an Axioskop inverted microscope with an AxioVision camera and software (Zeiss, Thornwood, NY, USA).

\section{RNA extraction and quantitative reverse transcription polymerase chain reaction}

Total RNA was extracted from distal colon segments or from colon tumors using TRIzol (Invitrogen, Carlsbad, CA, USA) and QIAGEN RNAeasy isolation kits (QIAGEN, Valencia, CA, USA). qRT-PCR was performed in triplicate using $4 \mu \mathrm{L}$ of $1 / 12$ diluted cDNA and SYBR green (Bio-Rad \#1708886) in $20 \mu \mathrm{L}$ total volume on a Roche 480 (Roche, Basel, Switzerland). The mRNA level was calculated by the cycle threshold $(\mathrm{Ct})$ value and was normalized by glyceraldehyde 3-phosphate dehydrogenase (GAPDH).

\section{Detection of tumor mutations}

Tumor tissues were obtained by laser capture microdissection (LCM) from paraffin-embedded colons and were digested with proteinase K. Genomic DNA was purified by standard phenol chloroform extraction. Exon 3 of the $\beta$-catenin gene was amplified by PCR using the following specific primers: 5'-GCTGACCTGATGGAGTTGGA-3' and 5'- GCTACTT GCTCTTGCGTGAA-3' (amplicon size $=227$ bp). PCR products were purified and sequenced using forward and reverse primers. Mutations were detected by observing individual chromatograms.

\section{Statistical analysis}

Data are expressed as mean \pm standard error of the mean (SEM). Differences were analyzed by Student's $t$ test. Values of $p<0.05$ were considered significant.

\section{CONFLICTS OF INTEREST}

The authors declare no conflicts of interest.

\section{FUNDING}

This work was supported by a grant (No. K17281) from the Korea Institute of Oriental Medicine funded by the Ministry of Science, ICT, and Future Planning and the National Research Foundation of Korea (NRF) grant funded by the Ministry of Education, Science, and
Technology grants NRF-2017M3A9D8057887 and NRF2016M3A9D5A01952411 and by Basic Science Research Program through the National Research Foundation of Korea (NRF) funded by the Ministry of Education (NRF2017R1D1A1B03032284).

\section{REFERENCES}

1. Ullman TA, Itzkowitz SH. Intestinal inflammation and cancer. Gastroenterology. 2011; 140:1807-16. https://doi. org/10.1053/j.gastro.2011.01.057.

2. Fearon ER, Vogelstein B. A genetic model for colorectal tumorigenesis. Cell. 1990; 61:759-67.

3. Fearon ER. Molecular genetics of colorectal cancer. Annu Rev Pathol. 2011; 6:479-507. https://doi.org/10.1146/ annurev-pathol-011110-130235.

4. Hanahan D, Weinberg RA. Hallmarks of cancer: the next generation. Cell. 2011; 144:646-74. https://doi. org/10.1016/j.cell.2011.02.013.

5. Rogler G. Inflammatory bowel disease cancer risk, detection and surveillance. Dig Dis. 2012; 30:48-54. https://doi. org/10.1159/000341893.

6. Schetter AJ, Heegaard NH, Harris CC. Inflammation and cancer: interweaving microRNA, free radical, cytokine and p53 pathways. Carcinogenesis. 2010; 31:37-49. https://doi. org/10.1093/carcin/bgp272.

7. Mantovani A, Allavena P, Sica A, Balkwill F. Cancerrelated inflammation. Nature. 2008; 454:436-44. https:// doi.org/10.1038/nature07205.

8. Grivennikov SI, Wang K, Mucida D, Stewart CA, Schnabl B, Jauch D, Taniguchi K, Yu GY, Osterreicher CH, Hung KE, Datz C, Feng Y, Fearon ER, et al. Adenoma-linked barrier defects and microbial products drive IL-23/IL-17-mediated tumour growth. Nature. 2012; 491:254-8. https://doi. org/10.1038/nature11465.

9. Westbrook AM, Szakmary A, Schiestl RH. Mechanisms of intestinal inflammation and development of associated cancers: lessons learned from mouse models. Mutat Res. 2010; 705:40-59. https://doi.org/10.1016/j.mrrev. 2010.03.001.

10. Schmitz H, Barmeyer C, Fromm M, Runkel N, Foss HD, Bentzel CJ, Riecken EO, Schulzke JD. Altered tight junction structure contributes to the impaired epithelial barrier function in ulcerative colitis. Gastroenterology. 1999; 116:301-9.

11. Tomas NM, Masur K, Piecha JC, Niggemann B, Zanker KS. Akt and phospholipase Cgamma are involved in the regulation of growth and migration of MDA-MB-468 breast cancer and SW480 colon cancer cells when cultured with diabetogenic levels of glucose and insulin. BMC Res Notes. 2012; 5:214. https://doi.org/10.1186/1756-0500-5-214.

12. Yang YR, Follo MY, Cocco L, Suh PG. The physiological roles of primary phospholipase C. Adv Biol Regul. 2013; 53:232-41. https://doi.org/10.1016/j.jbior.2013.08.003. 
13. Kang JK, Chang CH, Nam HJ, Kim SK, Ahn KJ, Seok H, Park SJ, Kang YJ, Jo YS, Shong M, Kim H. Downregulation of erythropoietin receptor by overexpression of phospholipase C-gamma 1 is critical for decrease on focal adhesion in transformed cells. Cell Oncol (Dordr). 2011; 34:11-21. https://doi.org/10.1007/s13402-010-0001-9.

14. Tan L, Xiao BX, Zeng WS, Lin J, Zou ZP, Xu AM, Luo SQ. Antitumour effects on human colorectal carcinomas cells by stable silencing of phospholipase C-gamma 1 with lentivirus-delivered siRNA. Chin Med J (Engl). 2007; 120:749-54.

15. Pegg AE. Methylation of the $\mathrm{O} 6$ position of guanine in DNA is the most likely initiating event in carcinogenesis by methylating agents. Cancer Invest. 1984; 2:223-31.

16. Boivin GP, Washington K, Yang K, Ward JM, Pretlow TP, Russell R, Besselsen DG, Godfrey VL, Doetschman T, Dove WF, Pitot HC, Halberg RB, Itzkowitz SH, et al. Pathology of mouse models of intestinal cancer: consensus report and recommendations. Gastroenterology. 2003; 124:762-77. https://doi.org/10.1053/gast.2003.50094.

17. Okayasu I, Ohkusa T, Kajiura K, Kanno J, Sakamoto S. Promotion of colorectal neoplasia in experimental murine ulcerative colitis. Gut. 1996; 39:87-92.

18. Okayasu I, Hatakeyama S, Yamada M, Ohkusa T, Inagaki Y, Nakaya R. A novel method in the induction of reliable experimental acute and chronic ulcerative colitis in mice. Gastroenterology. 1990; 98:694-702.

19. Morin PJ, Sparks AB, Korinek V, Barker N, Clevers H, Vogelstein B, Kinzler KW. Activation of beta-catenin-Tcf signaling in colon cancer by mutations in beta-catenin or APC. Science. 1997; 275:1787-90.

20. Bae SS, Perry DK, Oh YS, Choi JH, Galadari SH, Ghayur T, Ryu SH, Hannun YA, Suh PG. Proteolytic cleavage of phospholipase C-gamma1 during apoptosis in Molt-4 cells. FASEB J. 2000; 14:1083-92.

21. Liu X, Ye K. Src homology domains in phospholipase C-gammal mediate its anti-apoptotic action through regulating the enzymatic activity. J Neurochem. 2005; 93:892-8. https://doi.org/10.1111/j.1471-4159.2005.03064.x.

22. Toft NJ, Winton DJ, Kelly J, Howard LA, Dekker M, te Riele H, Arends MJ, Wyllie AH, Margison GP, Clarke AR. Msh2 status modulates both apoptosis and mutation frequency in the murine small intestine. Proc Natl Acad Sci U S A. 1999; 96:3911-5.

23. Ventura JJ, Tenbaum S, Perdiguero E, Huth M, Guerra C, Barbacid M, Pasparakis M, Nebreda AR. p38alpha MAP kinase is essential in lung stem and progenitor cell proliferation and differentiation. Nat Genet. 2007; 39:750-8. https://doi.org/10.1038/ng2037.

24. Smith MR, Liu YL, Matthews NT, Rhee SG, Sung WK, Kung HF. Phospholipase C-gamma 1 can induce DNA synthesis by a mechanism independent of its lipase activity. Proc Natl Acad Sci USA. 1994; 91:6554-8.
25. Huang PS, Davis L, Huber H, Goodhart PJ, Wegrzyn RE, Oliff A, Heimbrook DC. An SH3 domain is required for the mitogenic activity of microinjected phospholipase C-gamma 1. FEBS Lett. 1995; 358:287-92.

26. Kim BY, Kang DO, Oh WK, Kim JH, Choi YK, Jang JS, Suh PG, Ryu SH, Mheen TI, Ahn JS. Involvement of $\mathrm{SH} 2-\mathrm{SH} 2-\mathrm{SH} 3$ domain of phospholipase cgamma1 in NF-kappaB signaling. FEBS Lett. 2000; 472:45-9.

27. De Robertis M, Massi E, Poeta ML, Carotti S, Morini S, Cecchetelli L, Signori E, Fazio VM. The AOM/DSS murine model for the study of colon carcinogenesis: From pathways to diagnosis and therapy studies. J Carcinog. 2011; 10:9. https://doi.org/10.4103/1477-3163.78279.

28. Lee YH, Bae SS, Seo JK, Choi I, Ryu SH, Suh PG. Interleukin-6-induced tyrosine phosphorylation of phospholipase C-gamma1 in PC12 cells. Mol Cells. 2000; 10:469-74.

29. Choi JH, Kim HS, Kim SH, Yang YR, Bae YS, Chang JS, Kwon HM, Ryu SH, Suh PG. Phospholipase Cgamma1 negatively regulates growth hormone signalling by forming a ternary complex with Jak2 and protein tyrosine phosphatase-1B. Nat Cell Biol. 2006; 8:1389-97. https:// doi.org/10.1038/ncb1509.

30. Grivennikov S, Karin M. Autocrine IL-6 signaling: a key event in tumorigenesis? Cancer Cell. 2008; 13:7-9. https:// doi.org/10.1016/j.ccr.2007.12.020.

31. Iwamoto M, Koji T, Makiyama K, Kobayashi N, Nakane PK. Apoptosis of crypt epithelial cells in ulcerative colitis. J Pathol. 1996; 180:152-9. https://doi. org/10.1002/(SICI)1096-9896(199610)180:2<152::AIDPATH649>3.0.CO;2-Y.

32. Atreya R, Neurath MF. Involvement of IL-6 in the pathogenesis of inflammatory bowel disease and colon cancer. Clin Rev Allergy Immunol. 2005; 28:187-96. https://doi.org/10.1385/CRIAI:28:3:187.

33. Mudter J, Neurath MF. Il-6 signaling in inflammatory bowel disease: pathophysiological role and clinical relevance. Inflamm Bowel Dis. 2007; 13:1016-23. https://doi. org/10.1002/ibd.20148.

34. Gonzalez-Mariscal L, Betanzos A, Nava P, Jaramillo BE. Tight junction proteins. Prog Biophys Mol Biol. 2003; 81:1-44.

35. Ward PD, Klein RR, Troutman MD, Desai S, Thakker DR. Phospholipase C-gamma modulates epithelial tight junction permeability through hyperphosphorylation of tight junction proteins. Journal of Biological Chemistry. 2002; 277:35760-5. https://doi.org/10.1074/jbc.M203134200.

36. Chen ML, Pothoulakis C, LaMont JT. Protein kinase $\mathrm{C}$ signaling regulates ZO-1 translocation and increased paracellular flux of T84 colonocytes exposed to Clostridium difficile toxin A. J Biol Chem. 2002; 277:4247-54. https:// doi.org/10.1074/jbc.M109254200.

37. Cosnes J, Gower-Rousseau C, Seksik P, Cortot A. Epidemiology and natural history of inflammatory bowel 
diseases. Gastroenterology. 2011; 140:1785-94. https://doi. org/10.1053/j.gastro.2011.01.055.

38. Haggar FA, Boushey RP. Colorectal cancer epidemiology: incidence, mortality, survival, and risk factors. Clin Colon Rectal Surg. 2009; 22:191-7. https://doi. org/10.1055/s-0029-1242458.

39. Falasca M, Logan SK, Lehto VP, Baccante G, Lemmon MA, Schlessinger J. Activation of phospholipase C gamma by PI 3-kinase-induced $\mathrm{PH}$ domain-mediated membrane targeting. EMBO J. 1998; 17:414-22. https:// doi.org/10.1093/emboj/17.2.414.

40. Sala G, Dituri F, Raimondi C, Previdi S, Maffucci T, Mazzoletti M, Rossi C, Iezzi M, Lattanzio R, Piantelli M, Iacobelli S, Broggini M, Falasca M. Phospholipase C gamma 1 Is Required for Metastasis Development and Progression. Cancer Research. 2008; 68:10187-96. https:// doi.org/10.1158/0008-5472.Can-08-1181.

41. Koesters R, Hans MA, Benner A, Prosst R, Boehm J, Gahlen J, Doeberitz MK. Predominant mutation of codon 41 of the beta-catenin proto-oncogene in rat colon tumors induced by 1,2-dimethylhydrazine using a complete carcinogenic protocol. Carcinogenesis. 2001; 22:1885-90.

42. Takahashi M, Wakabayashi K. Gene mutations and altered gene expression in azoxymethane-induced colon carcinogenesis in rodents. Cancer Sci. 2004; 95:475-80.

43. Corvinus FM, Orth C, Moriggl R, Tsareva SA, Wagner S, Pfitzner EB, Baus D, Kaufmann R, Huber LA, Zatloukal K, Beug H, Ohlschlager P, Schutz A, et al. Persistent STAT3 activation in colon cancer is associated with enhanced cell proliferation and tumor growth. Neoplasia. 2005; 7:545-55.

44. Lassmann S, Schuster I, Walch A, Gobel H, Jutting U, Makowiec F, Hopt U, Werner M. STAT3 mRNA and protein expression in colorectal cancer: effects on STAT3inducible targets linked to cell survival and proliferation. J Clin Pathol. 2007; 60:173-9. https://doi.org/10.1136/ jcp.2005.035113.

45. Galluzzi L, Larochette N, Zamzami N, Kroemer G. Mitochondria as therapeutic targets for cancer chemotherapy. Oncogene. 2006; 25:4812-30. https://doi. org/10.1038/sj.onc. 1209598.

46. June $\mathrm{CH}$, Fletcher MC, Ledbetter JA, Samelson LE. Increases in tyrosine phosphorylation are detectable before phospholipase $\mathrm{C}$ activation after $\mathrm{T}$ cell receptor stimulation. J Immunol. 1990; 144:1591-9.

47. Grivennikov S, Karin E, Terzic J, Mucida D, Yu GY, Vallabhapurapu S, Scheller J, Rose-John S, Cheroutre
H, Eckmann L, Karin M. IL-6 and Stat3 are required for survival of intestinal epithelial cells and development of colitis-associated cancer. Cancer Cell. 2009; 15:103-13. https://doi.org/10.1016/j.ccr.2009.01.001.

48. Zhang P, Zhao Y, Zhu X, Sedwick D, Zhang X, Wang Z. Cross-talk between phospho-STAT3 and PLCgamma1 plays a critical role in colorectal tumorigenesis. Mol Cancer Res. 2011; 9:1418-28. https://doi.org/10.1158/1541-7786. MCR-11-0147.

49. Kishimoto T. Interleukin-6: from basic science to medicine- -40 years in immunology. Annu Rev Immunol. 2005; 23:1-21. https://doi.org/10.1146/annurev.immunol.23.021704.115806.

50. Zaki MH, Vogel P, Malireddi RK, Body-Malapel M, Anand PK, Bertin J, Green DR, Lamkanfi M, Kanneganti TD. The NOD-like receptor NLRP12 attenuates colon inflammation and tumorigenesis. Cancer Cell. 2011; 20:649-60. https:// doi.org/10.1016/j.ccr.2011.10.022.

51. Diakonova M, Chilov D, Arnaoutov A, Alexeyev V, Nikolsky N, Medvedeva N. Intracellular distribution of phospholipase $\mathrm{C}$ gamma 1 in cell lines with different levels of transformation. Eur J Cell Biol. 1997; 73:360-7.

52. Emori Y, Sugaya R, Akimaru H, Higashijima S, Shishido E, Saigo K, Homma Y. Drosophila phospholipase $\mathrm{C}$-gamma expressed predominantly in blastoderm cells at cellularization and in endodermal cells during later embryonic stages. J Biol Chem. 1994; 269:19474-9.

53. Tai YH, Flick J, Levine SA, Madara JL, Sharp GW, Donowitz M. Regulation of tight junction resistance in T84 monolayers by elevation in intracellular $\mathrm{Ca} 2+$ : a protein kinase C effect. J Membr Biol. 1996; 149:71-9.

54. Nguyen TD, Carrascal M, Vidal-Cortes O, Gallardo O, Casas V, Gay M, Phan VC, Abian J. The phosphoproteome of human Jurkat $\mathrm{T}$ cell clones upon costimulation with anti-CD3/anti-CD28 antibodies. J Proteomics. 2016; 131:190-8. https://doi.org/10.1016/j.jprot.2015.10.029.

55. Van Itallie CM, Anderson JM. The molecular physiology of tight junction pores. Physiology (Bethesda). 2004; 19:331-8. https://doi.org/10.1152/physiol.00027.2004.

56. el Marjou F, Janssen KP, Chang BH, Li M, Hindie V, Chan L, Louvard D, Chambon P, Metzger D, Robine S. Tissuespecific and inducible Cre-mediated recombination in the gut epithelium. Genesis. 2004; 39:186-93. https://doi. org/10.1002/gene.20042. 